\title{
Integrated Modeling of Active and Passive Microwaves and Passive Optical Signatures
}

\author{
I. Baris ${ }^{1}$, T. Jagdhuber ${ }^{1}$, F. Jonard ${ }^{2,3}$, J. Judge ${ }^{4}$, H. Anglberger ${ }^{1}$ \\ ${ }^{1}$ German Aerospace Center, Microwaves and Radar Institute, P.O. Box 1116, 82234 Wessling \\ ${ }^{2}$ Forschungszentrum Jülich GmbH, Institute of Bio- and Geosciences: Agrosphere (IBG-3), 52425 Jülich, Germany \\ ${ }^{3}$ Université catholique de Louvain, Earth and Life Institute, Croix du Sud 2, 1348 Louvain-la-Neuve, Belgium \\ ${ }^{4}$ Center for Remote Sensing, Agricultural and Biological Engineering Department, Institute of Food and Agricultural Sciences, \\ University of Florida, Gainesville, USA \\ E-Mail: ismail.baris@dlr.de, thomas.jagdhuber@dlr.de,f.jonard@fz-juelich.de, jasmeet@ufl.edu, harald.anglberger@dlr.de
}

\begin{abstract}
A method of physical integration of scattering models is presented here to estimate the backscattering coefficient (BSC) for L-band, brightness temperature (TB) for L- and C-Band and the reflectance for visible (VIS) and near-infrared (NIR) region for dynamic vegetated terrain. The SPIN (Spectrum Invariant Interaction) model is obtained by solving vector radiative transfer (VRT) equations kernel-based and therefore for different wave interaction mechanisms. To demonstrate its application for the microwave region, the measurements during the growing cycle of corn from the Eleventh Microwave, Water, and Energy Balance Experiment (MicroWEX-11) have been used. For the optical part the results are compared with the PROSAIL model. By applying the SPIN model in the radar regime, it could be shown that the modeled backscattering coefficients (BSC) correlate strongly with the vertical polarization measurements (Pearson $0.83, R^{2} 0.69$ ) and are less correlated with the horizontal measurements (Pearson 0.45, $\mathrm{R}^{\mathbf{2}} \mathbf{0 . 2 0}$ ). In addition, the modeled brightness temperatures ( $\mathrm{L}$ - and $\mathrm{C}$-band) in both polarization states are also highly correlated with the MicroWEX-11 measurements (L-band: Pearson 0.755, $\mathrm{R}^{2}$ 0.57; C-band: Pearson $\left.0.73, R^{2} 0.53\right)$. Finally, the optical results are consistent with the results of other optical models (Pearson $0.99, R^{2} 0.98$ ).
\end{abstract}

Keywords—radiative transfer, microwave, radar, optics

\section{INTRODUCTION}

A unifying parametrization and spectrum-overarching modeling of vegetation and soil properties for prediction and analysis of wave interaction along the EM spectrum is becoming increasingly important, especially in the light of the increasing fleet of earth observation sensors. The differing parameterization and modeling of the electromagnetic wave interaction in remote sensing algorithms ends in different biophysical vitality/productivity parameters or moisture and structure characteristics modeled in different ways. Therefore, it is desirable to develop an integrated model on physical basis, which describes/parameterizes scattering and emission characteristics in terms of both optical and radar/microwave wavelengths. With this objective we developed a kernel-driven wave interaction model, called SPIN (Spectrum Invariant Interaction) model, that is flexible enough to model the different scattering and emission characteristics for microwaves and optics. The main advantage of a kernel-driven approach is its analytical invertibility.

\section{METHODS}

The modified Stokes vectors describe polarized electromagnetic waves. The total amount of the received intensity $I$ is a sum of all polarization states [1]

$$
I^{2}=\left(I_{v}^{2}-I_{h}^{2}\right)+U^{2}+V^{2}
$$

If the beam is a mixture of polarized and unpolarized waves, then (1) becomes an inequality [1]. Hence, the specific intensity can be decomposed in a polarized $\mathbf{I}_{P}$ and unpolarized $\mathbf{I}_{U}$ Stokes vector $[1,2]$

$$
\mathbf{I}=\mathbf{I}_{P}+\mathbf{I}_{U},
$$

with

$$
\begin{array}{cc}
I_{P}=\mathfrak{P} \cdot \sqrt{\left(I_{v}^{2}-I_{h}^{2}\right)+U^{2}+V^{2}} \quad 0 \leq \mathfrak{P} \leq 1, \\
I_{U}=(1-\mathfrak{P}) \cdot I=I-\left(\mathfrak{P} \cdot I_{P}\right) \quad 0 \leq \mathfrak{P} \leq 1,
\end{array}
$$

where $\mathfrak{P}$ is the degree of polarization (DOP). Furthermore, (4) implies, that if $\mathfrak{P}$ is equal to zero (perfect unpolarized) the total amount of intensity collapses to the scalar form of the intensity like $\mathbf{I}=I$. Notice, all bold letters are vectors or matrices.

Accordingly, one can express the radiation loss in a medium as [3]

$$
\mu \frac{\partial \mathbf{I}_{v}(z, \mu, \phi)}{\partial z}=-\kappa_{e v} \mathbf{I}_{v}(z, \mu, \phi)+\kappa_{a v} \mathbf{B}_{v}(T)+\kappa_{s v} \mathbf{J}_{v}(z, \mu, \phi),
$$

with $\mu=\cos \theta$ (viewing angle) and $v$ symbolizes the frequency dependence. The term $\kappa_{a v} \mathbf{B}_{v}(T)$ describes emission from the medium, where $\kappa_{a v}$ is the absorption coefficient and $T$ is the temperature of the medium [3]. The last term $\mathbf{J}_{v}(z, \mu, \phi)$ describes the energy increment due to the scattering processes where $\kappa_{s v}$ is the scattering coefficient [3]

$$
\mathbf{J}_{v}(z, \mu, \phi)=\int_{2 \pi} \mathbf{P}\left(\mu, \phi \mid \mu^{\prime}, \phi^{\prime}\right) \mathbf{I}_{v}\left(\mu, \phi \mid \mu^{\prime}, \phi^{\prime}\right) d \underline{\Omega}^{\prime},
$$



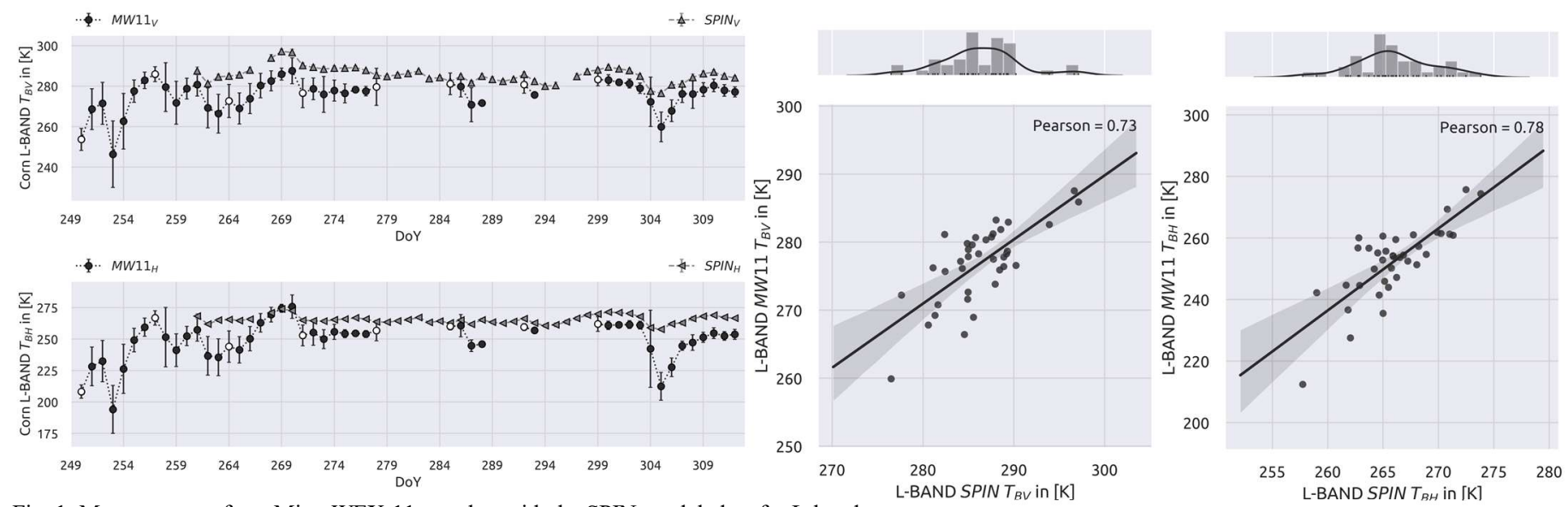

Fig. 1: Measurements from MicroWEX-11 together with the SPIN-modeled $T_{B}$ for L-band.

Left plots - X-axis: DoY in 2012. Top: Modeled and measured $T_{B}$ for vertical polarization. Bottom: Modeled and measured $T_{B}$ for horizontal polarization. White dots: In-situ measurements of plant parameters. Linear interpolation was performed between these points. Middle plot: The evaluation of the results for $T_{B V}$ with Pearson $0.73\left(\mathrm{R}^{2}=0.53, \mathrm{RMSE}=10.14\right)$. Right plot: The evaluation of the results for $T_{B H}$ with Pearson $0.78\left(\mathrm{R}^{2}=0.61, \mathrm{RSME}=16.52\right)$. The grey areas are the uncertainties calculated with different weightings of the residuals. The histogram and the density of the SPIN data is located above the middle and right graphics.

where $\mathbf{P}\left(\mu, \phi \mid \mu^{\prime}, \phi^{\prime}\right)$ is a $4 \times 4$ phase matrix that describes scattering directions of an incoming beam and $d \underline{\Omega}^{\prime}=\sin \theta^{\prime} d \theta^{\prime} d \phi^{\prime}$. Note, that the coefficient $\kappa_{a v}$ and $\kappa_{s v}$ are also $4 \times 4$ matrices. In the following sections, the notation $\mathbf{I}_{A v}^{+}(\mu, \phi, z)$ implies the solution of the VRT (Vector Radiative Transfer) for a bistatic case with scattering only (active) and $\mathbf{I}_{B v}^{+}(\mu, \phi, z)$ is the solution of the VRT for a monostatic case with emission and scattering (passive). $\mathbf{I}_{v}^{+}(\mu, \phi, z)$ is the total specific intensity. The "+" indicates the upward direction of the specific intensity.

As discussed in the previous section $\mathbf{I}_{v}^{+}(\mu, \phi, z)$ is composed of a polarized $\mathbf{I}_{v}^{P+}(\mu, \phi, z)$ and unpolarized $I_{v}^{U+}(\mu, \phi, z)$ Stokes vector. Thus, the total specific intensity of VRT changes to

$$
\mathbf{I}_{v}^{+}(\mu, \phi, z)=\left(\mathbf{I}_{v}^{P+}(\mu, \phi, z)+I_{v}^{U+}(\mu, \phi, z)\right) \cdot \mathbf{I}_{0} .
$$

As already stated in the introduction, it is desirable to represent the VRT equations as sum of linear equations, which will be called kernel-representation here. To achieve this, not all equations and polarizations $(\mathrm{HV}, \mathrm{VH})$ of first-order solutions can be considered yet. More investigation is still needed in order to consider the phase matrix at higher orders. Under these circumstances the active VRT solution leads to the following $2 \times 2$ kernel-representation for $\mathrm{VV}$ and $\mathrm{HH}$ polarization

$$
\mathbf{I}_{A v}^{P+}(\mu, \phi, z)=\boldsymbol{\beta}_{A 0}^{P}+\boldsymbol{\beta}_{A 1}^{P} \cdot \mathbf{K}_{A 1}^{P}+\boldsymbol{\beta}_{A 2}^{P} \cdot \mathbf{K}_{A 2}^{P},
$$

with

$$
\begin{aligned}
\mathbf{K}_{A 1}^{P}\left(\mu, \phi \mid-\mu_{0}, \phi_{0}\right) & =\frac{\mathbf{P}\left(\mu, \phi \mid-\mu_{0}, \phi_{0}\right)}{\mu+\mu_{0}}, \\
\mathbf{K}_{A 2}^{P}\left(\mu, \phi \mid \mu_{0}, \phi_{0}\right) & =\frac{\mathbf{P}\left(\mu, \phi \mid \mu_{0}, \phi_{0}\right)}{\mu_{0}-\mu},
\end{aligned}
$$

$$
\begin{gathered}
\boldsymbol{\beta}_{A 0}^{P}=\frac{\mathbf{w}_{v}^{P}}{\pi}+\gamma\left(\tau_{v}^{P}, \Omega\right)\left(\mathbf{R}^{P}\left(\mu_{0}\right)-\frac{\mathbf{w}_{v}^{P}}{\pi}\right), \\
\boldsymbol{\beta}_{A 1}^{P}=\mathbf{w}_{v}^{P} \cdot\left[1-\gamma\left(\tau_{v}^{P}, \Omega\right)\right] \mu, \\
\boldsymbol{\beta}_{A 2}^{P}=\mathbf{w}_{v}^{P} \cdot \mathbf{R}^{P}\left(\mu_{0}\right) \cdot\left[\gamma^{2}\left(\tau_{v}^{P}, \mu_{0}\right)-\gamma\left(\tau_{v}^{P}, \Omega\right)\right] \mu,
\end{gathered}
$$

where $\tau$ is the optical depth (if polarized a $2 \times 2$ matrix), $\mathbf{w}_{v}^{P}$ is the single scattering albedo, $\mathbf{R}^{P}$ is the bare soil reflectance, $\gamma\left(\tau_{v}^{P}, \mu_{x}\right)=\exp \left\{-\tau_{v}^{P} / \mu_{x}\right\}$ and $\gamma\left(\tau_{v}^{P}, \Omega\right)=\gamma\left(\tau_{v}^{P}, \mu_{0}\right) \cdot \gamma\left(\tau_{v}^{P}, \mu\right)$.

The solution for the unpolarized part of (7) is

$$
I_{A \nu}^{U+}(\mu, \phi, z)=F_{s t} \beta_{A 0}^{U}+\frac{2 \eta}{3 \pi} \beta_{A 1}^{U} \cdot K_{A 1}^{U}+\frac{2 \eta}{3 \pi} \beta_{A 2}^{U} \cdot K_{A 2}^{U},
$$

where the parameter $\beta_{A 0}^{U}, \beta_{A 1}^{U}, K_{A 1}^{U}, \beta_{A 2}^{U}$ and $K_{A 2}^{U}$ are described in equations (9) - (13). The parameter $F_{s t}=F_{s}+F_{t}$ is a function of $\mu$ as well as the leaf inclination which is multiplied by leaf reflectance to obtain volume scattering [4]. The parameter $\eta$ is defined as

$$
\eta=k_{e} \cdot F_{s} \cdot\left[1+\frac{F_{t} \cdot k_{t}}{F_{s} \cdot k_{s}}\right],
$$

where $k_{e}, k_{s}$ and $k_{t}$ are the extinction, scattering and transmission coefficients. Note, that the phase matrix in (9) and (10) must be exchanged with a phase function. The phase function is a scalar formulation of the phase matrix and does not take polarization in to account $[3,7]$.

Under the assumption of local thermal equilibrium (LTE) of the soil and vegetation the monostatic VRT with emission and scattering is obtained by

$$
\begin{aligned}
\mathbf{I}_{B \nu}^{P+}(\mu, \phi, z) & =\boldsymbol{\beta}_{B 0}^{P}+\boldsymbol{\beta}_{B 1}^{P}\left(\mu_{B 1}\right) \cdot \int_{2 \pi} \mathbf{K}_{A 1}^{P} d \underline{\Omega}, \\
& +\boldsymbol{\beta}_{B 2}^{P}\left(\mu_{B 2}\right) \cdot \int_{2 \pi} \mathbf{K}_{A 2}^{P} d \underline{\Omega},
\end{aligned},
$$



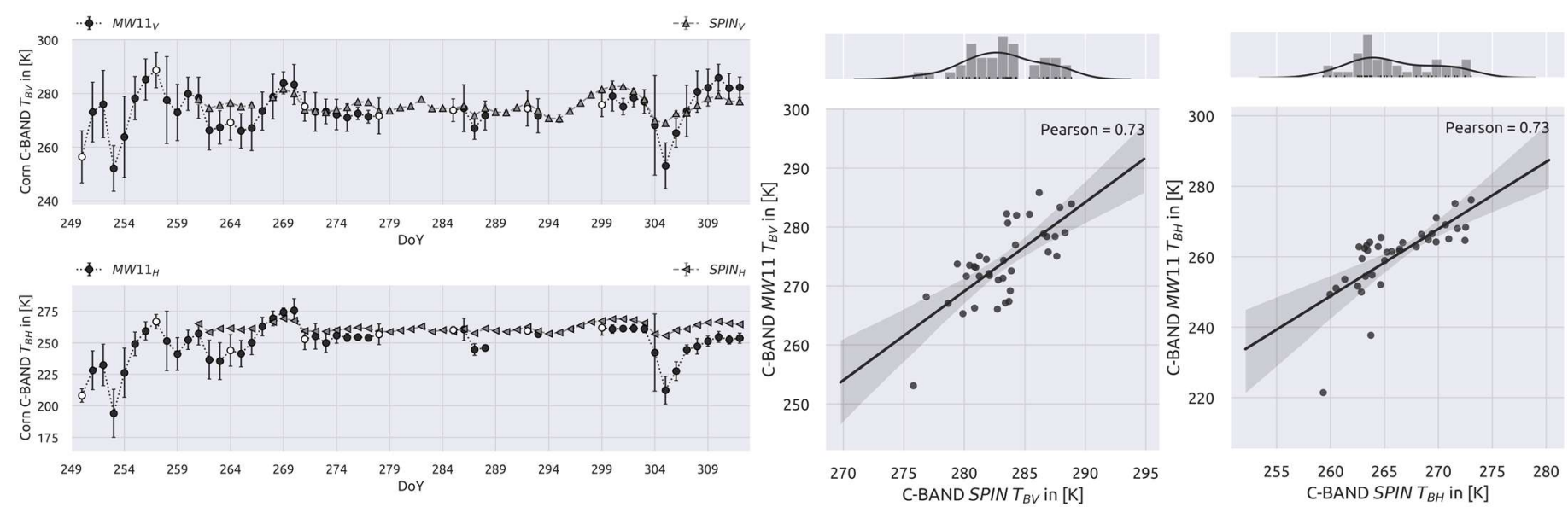

Fig. 2: Measurements from MicroWEX-11 together with the SPIN-modelled $T_{B}$ for C-band.

Left plots - X-axis: DoY in 2012. Top: Modelled and measured $T_{B}$ for vertical polarization. Bottom: Modelled and measured $T_{B}$ for horizontal polarization. White dots: In-situ measurements of plant parameters. Linear interpolation was performed between these points. Middle plot: The evaluation of the results for $T_{B V}$ with Pearson $0.73\left(\mathrm{R}^{2}=0.53\right.$, RMSE $\left.=9.49\right)$. Right plot: The evaluation of the results for $T_{B H}$ with Pearson $0.73\left(\mathrm{R}^{2}=0.53\right.$, RMSE=10.41). The grey areas are the uncertainties calculated with different weightings of the residuals. The histogram and the density of the SPIN data is located above the middle and right graphics. with

$$
\mathbf{I}_{A v}^{P^{+}}(\mu, \phi, z)=\mathbf{I}_{B v}^{P^{+}}(\mu, \phi, z) \cdot \Gamma_{A B v}^{+}(\mu, \phi, z)
$$

$$
\begin{aligned}
& \boldsymbol{\beta}_{B 0}^{P}= {\left[1-\gamma\left(\tau_{v}^{P}, \mu\right)\right]\left(1-\mathbf{w}_{v}^{P}\right) } \\
&+ \gamma\left(\tau_{v}^{P}, \mu\right) \cdot \mathbf{R}^{P}(\mu) \cdot\left[1-\gamma\left(\tau_{v}^{P}, \mu\right)\right]\left(1-\mathbf{w}_{v}^{P}\right), \\
&+\frac{\left(1-\mathbf{w}_{v}^{P}\right)}{\pi}+\gamma\left(\tau_{v}^{P}, \mu\right)\left(\left[1-\mathbf{R}^{P}(\mu)\right]-\frac{\left(1-\mathbf{w}_{v}^{P}\right)}{\pi}\right) \\
& \boldsymbol{\beta}_{B 1}^{P}\left(\mu_{B 1}\right)=\mathbf{w}_{v}^{P} \frac{\left(1-\mathbf{w}_{v}^{P}\left(\mu_{B 1}\right)\right)}{\kappa_{e v}\left(\mu_{B 1}\right)} \\
& \cdot\left[\gamma\left(\tau_{v}^{P}, \mu\right) \cdot\left(\mu_{B 1}+\mu\right)+\left\{\gamma\left(\tau_{v}^{P}, \Omega\right)-1\right\} \cdot \mu_{B 1}\right], \\
& \boldsymbol{\beta}_{B 2}^{P}\left(\mu_{B 2}\right)=\mathbf{w}_{v}^{P} \frac{\left(1-\mathbf{w}_{v}^{P}\left(\mu_{B 2}\right)\right)}{\kappa_{e v}\left(\mu_{B 2}\right)} \cdot\left[-\gamma\left(\tau_{v}^{P}, \mu\right) \cdot\left(\mu-2 \cdot \mu_{B 2}\right),\right. \\
&\left.+\gamma\left(\tau_{v}^{P}, \mu_{B 2}\right) \cdot \mu_{B 2}\right]
\end{aligned}
$$

and

$$
\mathbf{I}_{B v}^{P_{+}}(\mu, \phi, z)=\frac{\mathbf{I}_{A v}^{P^{+}}(\mu, \phi, z)}{\Gamma_{A B v}^{+}(\mu, \phi, z)} \rightarrow \mathbf{T}_{B v}(\mu, \phi, z)=\mathbf{I}_{B v}^{P_{+}}(\mu, \phi, z) \cdot T
$$

The angles $\mu_{B 1}$ and $\mu_{B 2}$ fulfill the following conditions

$$
\begin{aligned}
& \boldsymbol{\beta}_{B 1}^{P}\left(\mu_{B 1}\right) \cdot \int_{2 \pi} \mathbf{K}_{A 1}^{P} d \underline{\Omega}_{0}=\int_{2 \pi} \boldsymbol{\beta}_{B 1}^{P}\left(\mu_{0}\right) \cdot \mathbf{K}_{A 1}^{P} d \underline{\Omega}, \\
& \boldsymbol{\beta}_{B 2}^{P}\left(\mu_{B 2}\right) \cdot \int_{2 \pi} \mathbf{K}_{A 2}^{P} d \underline{\Omega}_{0}=\int_{2 \pi} \boldsymbol{\beta}_{B 2}^{P}\left(\mu_{0}\right) \cdot \mathbf{K}_{A 2}^{P} d \underline{\Omega} .
\end{aligned}
$$

The integration of (8) and (16) is obtained as follows

$$
\boldsymbol{\Gamma}_{A B v}^{+}(\mu, \phi, z)=\boldsymbol{\Gamma}_{0}+\boldsymbol{\Gamma}_{1} \cdot \mathbf{K}_{A B 1}+\boldsymbol{\Gamma}_{2} \cdot \mathbf{K}_{A B 2},
$$

with

$$
\begin{gathered}
\mathbf{K}_{A B x}=\frac{\mathbf{K}_{A x}^{P}}{\int_{2 \pi} \mathbf{K}_{A x}^{P} d \underline{\Omega}}, \\
\boldsymbol{\Gamma}_{x}=\frac{\boldsymbol{\beta}_{A x}^{P}}{\boldsymbol{\beta}_{B x}^{P}},
\end{gathered}
$$

With equation (22) it is now possible to describe $\mathbf{I}_{A v}^{P+}$ by $\mathbf{I}_{B v}^{P+}$ and vice versa:

\section{DATA}

MicroWEX-11 was a season-long experiment in north central Florida to monitor the microwave signatures of soil and sweet corn during different stages of growth [5]. The experiment was conducted from DoY (Day of Year) 250 until DoY 312 in 2012 with $T_{B}$ measurements at $1.4 \mathrm{GHz}$ (L-band) and $6.7 \mathrm{GHz}$ (C- Band) and BSC measurements at $1.25 \mathrm{GHz}$ (L-band) in 15 minutes intervals, as well as the soil measurements included volumetric soil moisture content (VSM) and soil temperature. These temperature measurements were used for equation (26) to obtain $T_{B}$. Vegetation sampling was conducted every week (8 measurements during the season) that included gravimetric water content of the plants (GWC), plant density, axis ratio of the plant, vegetation water content (VWC), leaf area index (LAI) and volume equivalent radius (see white dots in Fig. 1 - Fig. 4). These 8 measurements were linearly interpolated. The surface roughness were messearued at the beginning of the season.

\section{RESULTS AND DISCUSSION}

To obtain the phase, extinction and scattering matrix, the TMatrix method is used [6]. The phase function for the unpolarized part is from [7] and the extinction, scattering and transmission coefficients are obtained by the PROSPECT model [8]. For $\mathbf{I}_{A v}^{P+}(\mu, \phi, z)$ the $\mathrm{I}^{2} \mathrm{EM}$ and for $\mathbf{I}_{B v}^{P+}(\mu, \phi, z)$ the Fresnel reflection and for $I_{A v}^{U+}(\mu, \phi, z)$ a linear spectral mixing model is used to model the soil reflectance $[7,9]$.

Fig.1 visualizes the results of $T_{B}$ for L-band. Here, the modeled values are strongly correlated with the MicroWEX-11 measurements, although the modeled values range slightly too 

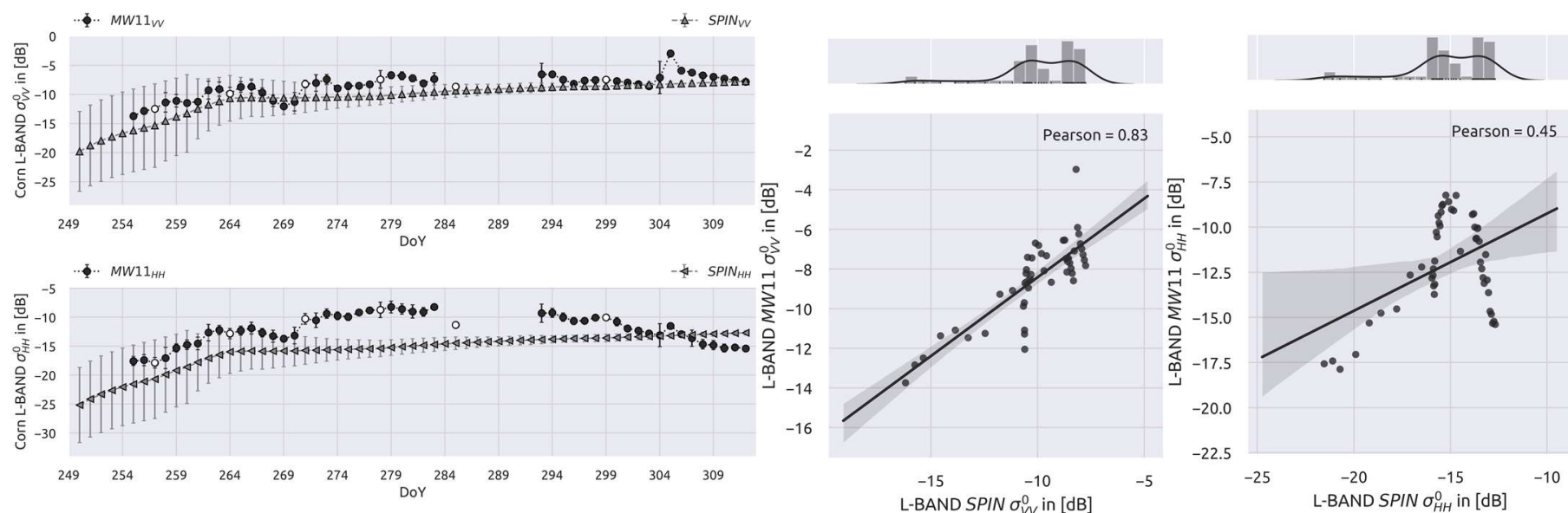

Fig.3: Measurements from MicroWEX-11 together with the SPIN-modelled BSC for L-band.

Left plots - X-axis: DoY in 2012. Top: Modelled and measured BSC for vertical polarization. Bottom: Modelled and measured BSC for horizontal polarization. White dots: In-situ measurements of plant parameters. Linear interpolation was performed between these points. Middle plot: The evaluation of the results for $\sigma_{V V}^{0}$ with Pearson $0.83\left(\mathrm{R}^{2}=0.69, \mathrm{RMSE}=2.01\right)$. Right plot: The evaluation of the results for $\sigma_{H H}^{0}$ with Pearson $0.45\left(\mathrm{R}^{2}=0.20, \mathrm{RMSE}=4.08\right)$. The grey areas are the uncertainties calculated with different weightings of the residuals. The histogram and the density of the SPIN data is located above the middle and right graphics.

high ( $T_{B V}$ - Pearson 0.73, $\mathrm{R}^{2}=0.53 ; T_{B H}$ - Pearson 0.78, $\left.\mathrm{R}^{2}=0.61\right)$. Like the modeled $T_{B}$ for C-band and BSC for L-band, this may be due to the modeling of soil reflections with the different soil models, which has caused some problems. For example, the modeled soil reflectance was much higher than the measured values. This is observable as before DoY 269, where the vegetation is not yet dominant, the modeled values are less accurate than after DoY 269.

The results of $T_{B}$ for C-band are shown in Fig. 2. Again, high correlations with the actual measurements are given (Pearson $0.73, \mathrm{R}^{2}=0.53$ ). Like with the results for L-band, also here the values after DoY 269 are more accurate than before DoY 269.

The modeled and measured BSC values are visualized in Fig. 3 . In the active case there are no continuously measured parameters like $T$ in (26). Thus, the dynamics of the model results are not pronounced due to the absence of continuously
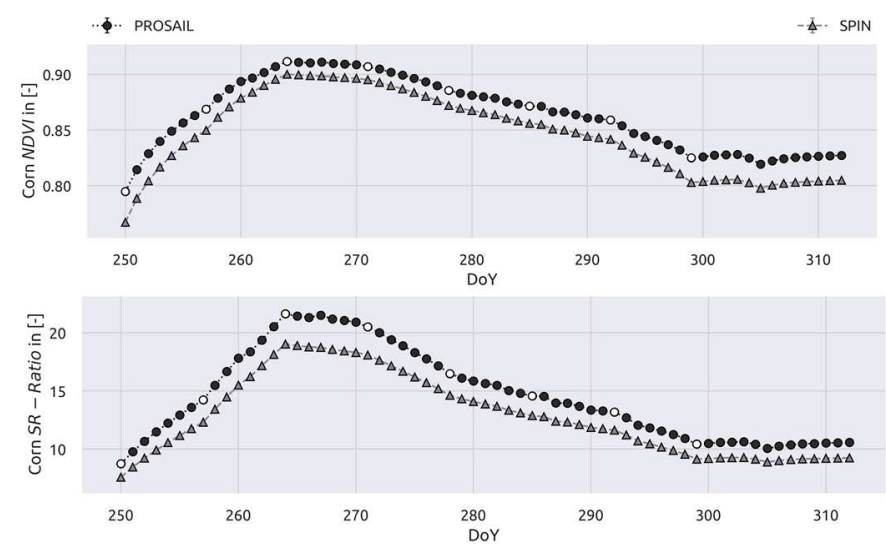

Fig.4: Experimental NDVI (top) and NIR-Red Ratio (SR, bottom) indices of SPIN and PROSAIL model.

Parameter: Leaf angle $=45^{\circ}$, Leaf structure parameter $=1$. Chlorophyll $\mathrm{a}+\mathrm{b}$ content $=20$. Carotenoids content $=3$. Brown pigments content $=0.40$. Equivalent water thickness. $=0.0005$. Dry matter content $=0.0085$. No hotspot parameter is considered. NDVI - Pearson $=0.99, \mathrm{R}^{2}=0,98$, RMSE $=0.018$. SR Pearson $=0.99, \mathrm{R}^{2}=0.98, \mathrm{RMSE}=1.82$. recorded data. The results show that the modeled VV polarization is much more accurate (Pearson 0.83, $\mathrm{R}^{2} 0.69$ ) than the $\mathrm{HH}$ polarization (Pearson $0.45, \mathrm{R}^{2} 0.20$.). This will be further investigated.

Fig. 4 shows the experimental results for the optical wavelength. Since no data is available for the validation of the optical region, these results are only of experimental nature. Therefore, only the LAI and VSM values were used to form the indices NDVI (Normalized Difference Vegetation Index) and SR (NIR-Red Ratio). As one can see the SPIN model has a very similar dynamic range as the PROSAIL model. One difference is, that the PROSAIL model generates higher values.

\section{ACKNOWLEDGEMENT}

We are grateful to MIT for supporting this research with the MIT-Germany Seed Fund "Global Water Cycle and Environmental Monitoring using Active and Passive Satellite-based Microwave Instruments" and with the MIT-Belgium UCL Seed Fund „Early Detection of Plant Water Stress Using Remote Sensing”.

\section{REFERENCES}

[1] M. Bass, Ed., Handbook of optics: VOLUME I: Fundamentals, Techniques and Design, 2nd ed. New York, NY: McGraw-Hill, 1995.

[2] C. F. Bohren and D. R. Huffman, Absorption and scattering of light by small particles. Weinheim: Wiley-VCH, 2007.

[3] S. Chandrasekhar, Radiative transfer. New York: Dover Publications, 1960.

[4] W. Verhoef, Theory of radiative transfer models applied in optical remote sensing of vegetation canopys. AD Emmerloord: National Aerospace Laboratory (NLR), 1998.

[5] UF IFAS Extension, University of FLORIDA "Field Observations during the Eleventh Microwave Water and Energy Balance Experiment (MicroWEX-11): from April 25, 2012, through December 6, 2012: AE514,” 2015.

[6] M. I. Mishchenko, L. D. Travis, and D. W. Mackowski, "T-matrix computations of light scattering by nonspherical particles: A review," Journal of Quantitative Spectroscopy and Radiative Transfer, vol. 55, no. 5, pp. 535-575, 1996.

[7] J. Ross, The radiation regime and architecture of plant stands. The Hague: Junk, 1981.

[8] S. Jacquemoud and F. Baret, "PROSPECT: A model of leaf optical properties spectra," Remote Sensing of Environment, vol. 34, no. 2, pp. 75-91, 1990.

[9] A. K. Fung, W. Y. Liu, K. S. Chen, and M. K. Tsay, "An Improved Iem Model for Bistatic Scattering From Rough Surfaces," Journal of Electromagnetic Waves and Applications, vol. 16, no. 5, pp. 689-702, 2002 